\title{
The geographic scaling of biotic interactions
}

\author{
Miguel B. Araújo and Alejandro Rozenfeld
}

M. B. Araújo (miguel.araujo@imperial.ac.uk), Imperial College London, Silwood Park Campus, Buckhurst Road, Ascot SL5 7PY, Berks, UK, and Dept of Biogeography and Global Change, National Museum of Natural Sciences, CSIC, Calle José Gutiérrez Abascal, 2, ES-28006, Madrid, Spain, and Center for Macroecology, Evolution and Climate, Univ. of Copenhagen, 2100 Copenhagen, Universitetsparken 15, DK-2100, Denmark. - A. Rozenfeld and MBA, InBio/CIBIO, Univ. of Évora, Largo dos Colegiais, PT-7000 Evora, Portugal.

\begin{abstract}
A central tenet of ecology and biogeography is that the broad outlines of species ranges are determined by climate, whereas the effects of biotic interactions are manifested at local scales. While the first proposition is supported by ample evidence, the second is still a matter of controversy. To address this question, we develop a mathematical model that predicts the spatial overlap, i.e. co-occurrence, between pairs of species subject to all possible types of interactions. We then identify the scale of resolution in which predicted range overlaps are lost. We found that co-occurrence arising from positive interactions, such as mutualism $(+/+)$ and commensalism $(+/ 0)$, are manifested across scales. Negative interactions, such as competition $(-/-)$ and amensalism $(-/ 0)$, generate checkerboard patterns of co-occurrence that are discernible at finer resolutions but that are lost and increasing scales of resolution. Scale dependence in consumer-resource interactions $(+/-)$ depends on the strength of positive dependencies between species. If the net positive effect is greater than the net negative effect, then interactions scale up similarly to positive interactions. Our results challenge the widely held view that climate alone is sufficient to characterize species distributions at broad scales, but also demonstrate that the spatial signature of competition is unlikely to be discernible beyond local and regional scales.
\end{abstract}

The question of whether the geographical ranges of species are determined by their ecological requirements and the physical characteristics of individual sites, or by assembly rules reflecting interactions between species, has long been a central issue in ecology (Andrewartha and Birch 1954, Diamond 1975, Gotelli and Graves 1996, Chase and Leibold 2003, Peterson et al. 2011). Evidence is compelling that the limits of species ranges often match combinations of climate variables, especially at high latitudes and altitudes (Grinnell 1917, Andrewartha and Birch 1954, Hutchinson 1957, Woodward 1987, Root 1988), and that these limits shift through time in synchrony with changes in climate (Walther et al. 2005, Hickling et al. 2006, Lenoir et al. 2008). However, recent evidence suggests that the thermal component of species climatic (fundamental) niches is more similar among terrestrial organisms than typically expected (Araújo et al. 2013), leading to the conclusion that spatial turnover among distributions of species might often result from non-climatic factors (see also for discussion Baselga et al. 2012a). The degree to which non-climatic factors shape the distributions of species has been focus of discussion in community ecology and biogeography for

This is an open access article under the terms of the Creative Commons Attribution License, which permits use, distribution and reproduction in any medium, provided the original work is properly cited. over a century, with several authors proposing that climate exerts limited influence at lower latitudes and altitudes (Wallace 1878, Dobzhansky 1950, Loehle 1998, Svenning and Skov 2004, Colwell et al. 2008, Baselga et al. 2012b). Specifically, much interest exists regarding the extent to which occurrences of species are constrained by the distributions of other species at broad scales of resolution and extent (Gravel et al. 2011). It has been argued that biotic interactions determine whether species thrives or withers in a given environment, but that the spatial effects associated with these interactions are lost at broad scales (Whittaker et al. 2001, Pearson and Dawson 2003, McGill 2010). In contrast, modelling studies have hinted that biotic interactions could leave broad-scale imprints on coexistence and, therefore, on species distributions (Anderson et al. 2002, Araújo and Luoto 2007, Heikkinen et al. 2007, Meier et al. 2010, Bateman et al. 2012, Madon et al. 2013). But empirical evidence for broad scale effects of biotic interactions is limited. A study has shown that with scales of few hundred kilometres the effects of competition on geographical ranges can still be discernible (Gotelli et al. 2010), but at scales of biomes such effects are often diluted (Russell et al. 2006, Veech 2006). How general are these patterns?

Empirical studies of the effects of biotic interactions on species distributions have historically focused on competition (Gause 1934, Hardin 1960, MacArthur 1972, 
Schoener 1982, Amarasekare 2003). However, several authors have pointed out that a greater variety of interactions can control for spatial patterns of overlap between species (Hairston et al. 1960, Connell 1975, Ricklefs 1987, 2010, Callaway et al. 2002, Bruno et al. 2003, Travis et al. 2005). Competition is a specific case involving two species that are worse off interacting with one another (which we annotate as: $-/-$ ). In its extreme form, competition leads to co-exclusion of the interacting species (MacArthur 1972). The reverse of competition is mutualism, whereby two species display mutual dependency $(+/+)$. Different combinations of positive, negative, and neutral relationships exist and they generate consumer-resource interactions such as predation, herbivory, parasitism and disease $(+/-)$, or amensalism $(-/ 0)$ and commensalism $(+/ 0)$.

The spatial effects of the different biotic interactions have rarely, if ever, been investigated. Differences in co-occurrence arising from alternative biotic interactions are seldom stated and focus has been on identifying non-random patterns of co-occurrence between pairs of species (Gotelli and McCabe 2002, Horner-Devine et al. 2007, Gotelli et al. 2010). Substantial controversy exists regarding the appropriate null models in such analyses (for review and discussion see Gotelli and Graves 1996), but the more fundamental question of whether departures from randomness in cooccurrence provide interpretable information regarding the underlying biotic interactions remains unanswered.

In practice, several biotic and abiotic factors can simultaneously affect the distributions of species (Soberón 2010, Peterson et al. 2011) and, therefore, co-occurrence (Cohen 1971, Leibold 1997, Amarasekare et al. 2004, Ovaskainen et al. 2010, Araújo et al. 2011). One approach to disentangle the relative importance of factors causing changes in species co-occurrence is through simulations (Urban 2005). Here, we develop a new 'point-process' model that infers co-occurrence of species at steady state across the full space of direct potential biotic interactions between pairs of species: i.e. given all biotic interaction types $(+/+,+/-,-/-,+/ 0,-/ 0)$ and all possible combinations of biotic interaction strength $\left(0 \leq I_{x} \leq 1\right)$. Dynamic Lotka-Volterra-type models can also be used as they explicitly simulate the effects of different biotic interactions on population dynamics (e.g. predation, competition, mutualism, see for review Kot 2001). However, Lotka-Volterra models require detailed parameterization of mortality and colonization rates that are highly contingent and are usually impossible to obtain. Furthermore, models predicting the spatial effects of repulsive and attractive interactions at steady state are particularly appropriate if the goal is to examine the effective spatial effects of interactions rather than the underlying population dynamics that generate them (see also Dieckmann et al. 2000, Law and Dieckmann 2000). The critical issue is whether a simple point-process model, such as ours, simulates spatial patterns of co-occurrence comparable with dynamic Lotka-Volterra models at equilibrium. Preliminary analysis comparing our model with Markov-chain formulation of Lotka-Volterra models by Cohen (1970) supports this view (Rozenfeld and Araújo unpubl.).

In the current implantation of our point-process model, and to control for the effects of species range sizes and environmental clustering on species distributions, simulations were replicated for species ranges with varying prevalence and spatial autocorrelation. Once co-occurrence between two species was estimated, we sampled ranges at increasingly coarser scales of resolution (i.e. by increasing grid-cell size) and identified the scale at which the original patterns of co-occurrences lost the spatial signature of the biotic interactions effects. When the effects of biotic interactions on patterns of co-occurrence of species were maintained across scales of resolution we interpreted the pattern as providing evidence for scale independence. In contrast, biotic interactions generating patterns of cooccurrence that were lost at increasing scales of resolutions were interpreted as being strongly dependent on the scale.

\section{Material and methods}

\section{The model}

The primary assumption of our point-process model is that the signal of biotic interactions drives spatial attraction $($ for + ) or repulsion (for - ). It follows that if no interactions are present $(0 / 0)$, co-occurrence between species ranges is dependent on their prevalence ( $\rho=$ fraction of the sites where the species is present). Formally, if species probabilities of occurrence are equal to their respective prevalence, i.e. $P(A)=\rho_{A}$ and $P(B)=\rho_{B}$, then the probability of cooccurrence between ranges of two non-interacting species is given by

$P_{\text {Null }}(A \cap B)=\rho_{A} \rho_{B}$

The probability of co-occurrence is the expected fraction of sites where species co-occur. If species A and B interact, then their overlap is a function of both their prevalence and the strength of their interactions $I_{A}$ and $I_{B}$

$P(A \cap B)=f\left(\rho_{A}, \rho_{B}, I_{A}, I_{B}\right)$

Interactions can be either attractive $I_{x}^{+}$or repulsive $I_{x}^{-}$, with $0 \leq I_{A}^{+}$or ${ }_{B}{ }_{B} \leq 1$. It follows that $I_{A}^{+}$stands for the intensity with which species $\mathrm{A}$ is attracted by $\mathrm{B}$, and $I_{B}^{+}$is the intensity with which species B is attracted by A. Likewise, $I_{A}^{-}$stands for the intensity with which species A repulses $\mathrm{B}$, and $I_{B}^{-}$is the intensity with which species $\mathrm{B}$ repulses A.

In the particular case of mutualism $(+/+)$, positive interactions will cause species to co-occur more often than expected under the null model

$P_{(+++)}(A \cap B)=\rho_{A} \rho_{B}+\max \left(I_{A}^{+}, I_{B}^{+}\right) \times\left[\min \left(\rho_{A}, \rho_{B}\right)-\rho_{A} \rho_{B}\right]$

Where the second term in Eq. 3 estimates the excess of cooccurrence due to positive $(+/+)$ interactions. The maximum fraction of sites where species co-occur is limited by the prevalence of the species with the most restricted range, i.e. $\min \left(\rho_{A}, \rho_{B}\right)$. So that $\left[\min \left(\rho_{A}, \rho_{B}\right)-\rho_{A} \rho_{B}\right]$ refers to the maximum excess of co-occurrence over the null model. With interactions $(+/+)$, the species with the greatest positive dependence is the one that constrains co-occurrence between the two interacting species. That is, the maximum excess of co-occurrence is modulated by the maximum attracting index $\left(\max \left(I_{A}^{+}, I_{B}^{+}\right)\right)$. 
So,

$P_{(+++)}(A \cap B)=\left\{\begin{array}{cccc}\rho_{A} \rho_{B} & I_{A}^{+}=0 & \text { and } & I_{B}^{+}=0 \\ \min \left(\rho_{A}, \rho_{B}\right) & I_{A}^{+}=1 & \text { or } & I_{B}^{+}=1\end{array}\right.$

When both interaction strengths are 0 we recover the null expectation, and when one of the species is fully dependent on the other the co-occurrence range is maximal.

In the case of competition $(-/-)$, negative interactions will cause the species to co-occur less often than expected under the null model

$P_{(-/-)}(A \cap B)=\rho_{A} \rho_{B} \times\left[1-\max \left(I_{A}^{-}, I_{B}^{-}\right)\right]$

Co-occurrence will tend to zero as the interaction strength of at least one of the interacting species approaches 1 . With interactions $(-/-)$, the species with the greatest negative (repulsive) interaction is the one that constrains co-occurrence between the two interacting species. That is, co-occurrence decreases below the null expectation proportionally to the maximum repulsion strength $\left(\max \left(I_{A}^{-}, I_{B}^{-}\right)\right)$.

So,

$P_{(-1-)}(A \cap B)=\left\{\begin{array}{cccc}\rho_{A} \rho_{B} & I_{A}^{-}=0 & \text { and } & I_{B}^{-}=0 \\ 0 & I_{A}^{-}=1 & \text { or } & I_{B}^{-}=1\end{array}\right.$

When both interaction strengths are 0 we recover the null expectation and, when one of the species is fully excluded by the other, co-occurrence is zero.

In the case of consumer-resource interactions $(+/-)$ with $\mathrm{A}$ being the consumer and $\mathrm{B}$ the resource, both positive and negative interactions will cause co-occurrence to deviates from the null expectation

$P_{(+/-)}(A \cap B)=\left[\rho_{A} \rho_{B}+I_{A}^{+} \times\left(\min \left(\rho_{A}, \rho_{B}\right)-\rho_{A} \rho_{B}\right)\right] \times\left(1-I_{B}^{-}\right]$

The Eq. 5 is a combination of Eq. 3 and 4. The first factor $\left[\rho_{A} \rho_{B}+\left(\min \left(\rho_{A}, \rho_{B}\right)-\rho_{A} \rho_{B}\right) I_{A}^{+}\right]$corresponds to Eq. 3 with $I_{B}^{+}=0$, and it shows how co-occurrence is increased due to the positive dependence of species $A$ on $B$. The second factor $\left(1-I_{B}^{-}\right)$reduces co-occurrence proportionally to the repulsive strength $I_{B}^{-}$.

So,

$P_{(+/)}(A \cap B)=\left\{\begin{array}{ccc}\rho_{A} \rho_{B} & I_{A}^{+}=0 & \text { and } I_{B}^{-}=0 \\ \min \left(\rho_{A}, \rho_{B}\right) & I_{A}^{+}=1 \text { and } I_{B}^{-}=0 \\ 0 & I_{B}^{-}=1\end{array}\right.$

When both interaction strengths are 0 we recover the null expectation. When species $\mathrm{A}$ is fully dependent on $\mathrm{B}$ and species $\mathrm{B}$ does not repulse $\mathrm{A}$ then the co-occurrence reaches its maximum. Finally, when species $B$ repulses A with maximum intensity co-occurrence is forbidden.

Notice that commensalism is a special case of mutualism (with $I_{B}^{+}=0$ ) or predation, parasitism and disease (with $\left.I_{B}^{-}=0\right)$, while amensalism is a special case of competition (with $\left.I_{B}^{-}=0\right)$. By varying the sign $(+,-)$ and the strength $I_{x}(0 \leq x \leq 1)$, our model predicts range overlaps across the full biotic interaction space.

\section{Simulations}

The general formulation of our point-process model defines rules of attraction and repulsion among species subject to different biotic interaction types and strengths, but these interactions take place in non-heterogeneous landscapes where multiple drivers, in addition to interactions, can affect species ranges and, therefore, co-occurrence. Constraints to the general model can be added to take these drivers into account, such as varying the ecological niches of species (both in the sense of species affecting and being affected by the environment, Chase and Leibold 2003, Peterson et al. 2011), or dispersal (both in the sense of species having the ability to disperse and being prevented from it due to external barriers, Levin 1974, Pulliam 1988, Hanski 1998, Humphries and Parenti 1999). Here, we explore two features of species ranges that we deem relevant for studying the geographical scaling of biotic interactions. The first is prevalence $(\rho)$. In one implementation of the model, the prevalence of species is relatively low: each species occupies $10 \%$ of the studied region $(\rho=0.1)$. In the other implementation of the model species occupy $30 \%(\rho=0.3)$ of the studied region.

The second feature explored is the placement of ranges. In one implementation of the model, species $B$ is randomly located and species $\mathrm{A}$ is affected by species $\mathrm{B}$. Under this model, environmental conditions are assumed to be homogenous across the studied area as it would be expected if range overlaps were measured within a given habitat type. In such a scenario, species $\mathrm{B}$ can be found anywhere in geographical space and range overlaps between species A and B are solely determined by prevalence and the attractive and repulsive effects of interactions. In the modified model, the distribution of species B is spatially structured while species A is a function of species B. This implementation of the model simulates range overlaps when the distribution of one of the species is highly autocorrelated (Legendre 1993, Dormann et al. 2007). Such autocorrelation can arise because strong environmental gradients exist and act to constrain species ranges (as might often occur at biogeographical extents) and/or when dispersal, demographic or behavioural factors cause individuals to aggregate in specific portions of geographical space (as might often occur at local and landscape extents). All simulations were performed in lattices of $100 \times 100$ pixels. In order to account for stochastic differences in the placement of the ranges, simulations were repeated 1000 times. Details on the generation of random and spatially autocorrelated distributions are provided in the supporting online material, together with the Mat Lab computer code written by $A R$ and used to generate the species ranges (Supplementary material).

\section{Measuring spatial dependencies in biotic interactions}

To address the question of how co-occurrences emerging from different biotic interactions affect species distributions at different spatial resolutions we used a hierarchical framework (Allen and Starr 1982). We compared co-occurrence 
scores (measured as the ratio of the number of geographical cells where species $\mathrm{A}$ and $\mathrm{B}$ co-occur to the total number of occupied cells) at the original resolution used to fit all of our models (the cell in our lattice landscape) with cooccurrences measured at progressively larger scales of resolution. This hierarchical framework for scaling was achieved by increasing the size of the blocks where individuals occur, and then quantifying the resulting co-occurrence. The quantification of co-occurrence was done using two approaches. The first seeks to preserve information about the 'true' co-occurrence of species that exists within geographical blocks and counts species as co-occurring if, and only if, they co-occur within one or more cells within the larger block. The second emulates the traditional approach of 'sampling' species occurrences' data in biogeography and macroecology (Rahbek and Graves 2001, Araújo 2004, Whittaker et al. 2005, McPherson et al. 2006, Nogués-Bravo and Araújo 2006), and counts species as co-occurring if both species are present somewhere in the block regardless of whether they co-exist in the cells.

The 'true' and 'sampled' co-occurrence scores measured at the cell level are then plotted against progressively larger block sizes. The area between the curves representing the 'true' and 'sampled' co-occurrences between species $\mathrm{A}$ and $\mathrm{B}$, across the range of block sizes, provides a measure of scale dependence of co-occurrence patterns (Fig. 1). The greater the area between the two curves the more the effects of given biotic interaction on species' distributions depends on spatial resolution, and vice versa (Fig. 1). The area between the 'true' and the 'sampled' co-occurrences is calculated for the full set of possible biotic interactions that can arise from combining interactions of varying signs $(+,-)$ and strengths $I_{x}(0 \leq x \leq 1)$ and true cooccurrences are by definition more conservative than sampled ones.

\section{Results}

Although positive interactions generate range overlaps and negative interactions generate non-overlaps, equivalent degrees of overlap were recorded for species exposed to different types of biotic interactions (Fig. 2). For example, the spatial patterns of range overlap for commensalism $\left(I_{A}^{+}, I_{B}^{0}\right)$ can be identical to range overlaps arising from mutualistic interactions $\left(I_{A}^{+}, I_{B}^{+}\right)$(Fig. 1). Range overlaps from amensalism $\left(I_{A}^{-}, I_{B}^{0}\right)$ can also mach range overlaps from competition $\left(I_{A}^{-}, I_{B}^{-}\right)$. Patterns of range overlap from consumer-resource interactions $\left(I_{A}^{-}, I_{B}^{+}\right)$can be like that of any type of biotic interaction.

When data are sampled from the cell to progressively larger blocks, estimated co-occurrence between species increases until an asymptote of complete overlap is reached (Fig. 3). The difference between 'sampled' and 'true' co-occurrence (our metric of scale independence, Fig. 1) varies with the spatial resolution, but also with the signal and the strength of the biotic interactions (Fig. 3). The stronger the negative interactions, the more scale dependent local patterns of co-occurrence are; in contrast, the stronger the positive interactions the greater the scale independence. In the extreme case of obligate positive dependencies between species pairs, i.e. strong mutualism, no difference exists between 'sampled' and 'true' co-occurrence across spatial scales.

Co-occurrence patterns generated by consumerresource interactions are also discernible across spatial scales, when at least one of the interacting species has strong positive dependency on the other. The same qualitative trend is maintained when species prevalence and autocorrelation increases (Table 1). However, scale independence tends to increase when interacting species have higher prevalence and ranges have weak spatial autocorrelation structure (Table 1). Spatially autocorrelated ranges also generate higher variance in patterns of scale dependence, chiefly across competitive interaction space (Table 1, Supplementary material Appendix 5, Fig. A1).

\section{Discussion}

Inferring process from pattern across scales is a critical challenge for ecology, biogeography, as well as for other

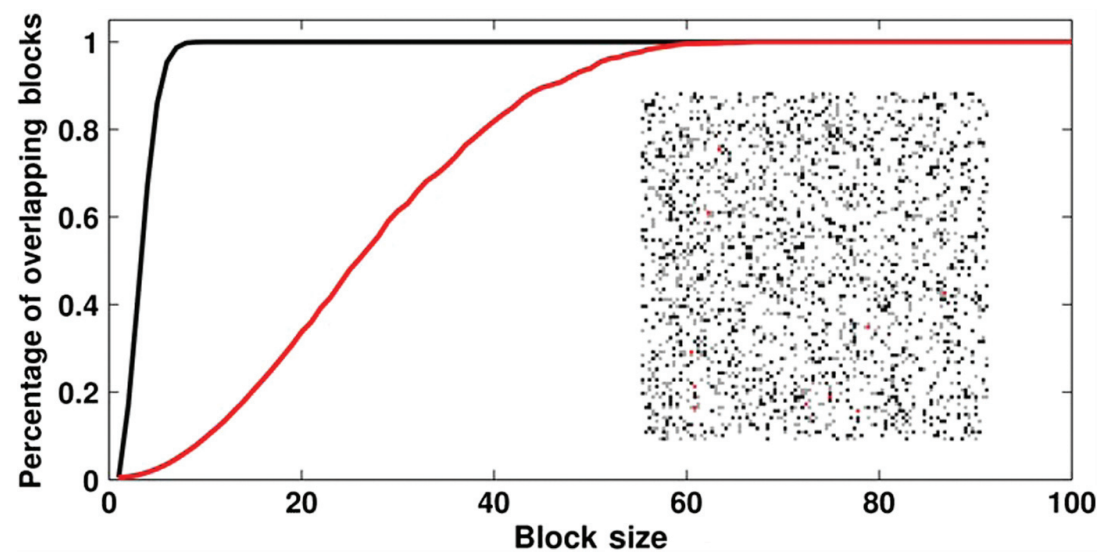

Figure 1. Scale dependence of biotic interactions. Right (squared landscape): after the range of species A and B have been simulated, co-occurrence between the two species is calculated. Black squares indicate occurrence of species A but not species B, gray squares indicate occurrence of B but not A, and red squares indicate co-occurrence of A and B. Left (diagram): by progressively increasing the size of the squares, 'sampling' leads to classifying species has co-occurring if both occur somewhere in the square (black line indicates 'resampled' co-occurrence), while true co-occurrence occurs when species overlap within the square (red line indicates 'true' co-occurrence). The greater the area between the red and black lines the greater the scale dependence of biotic interactions. 


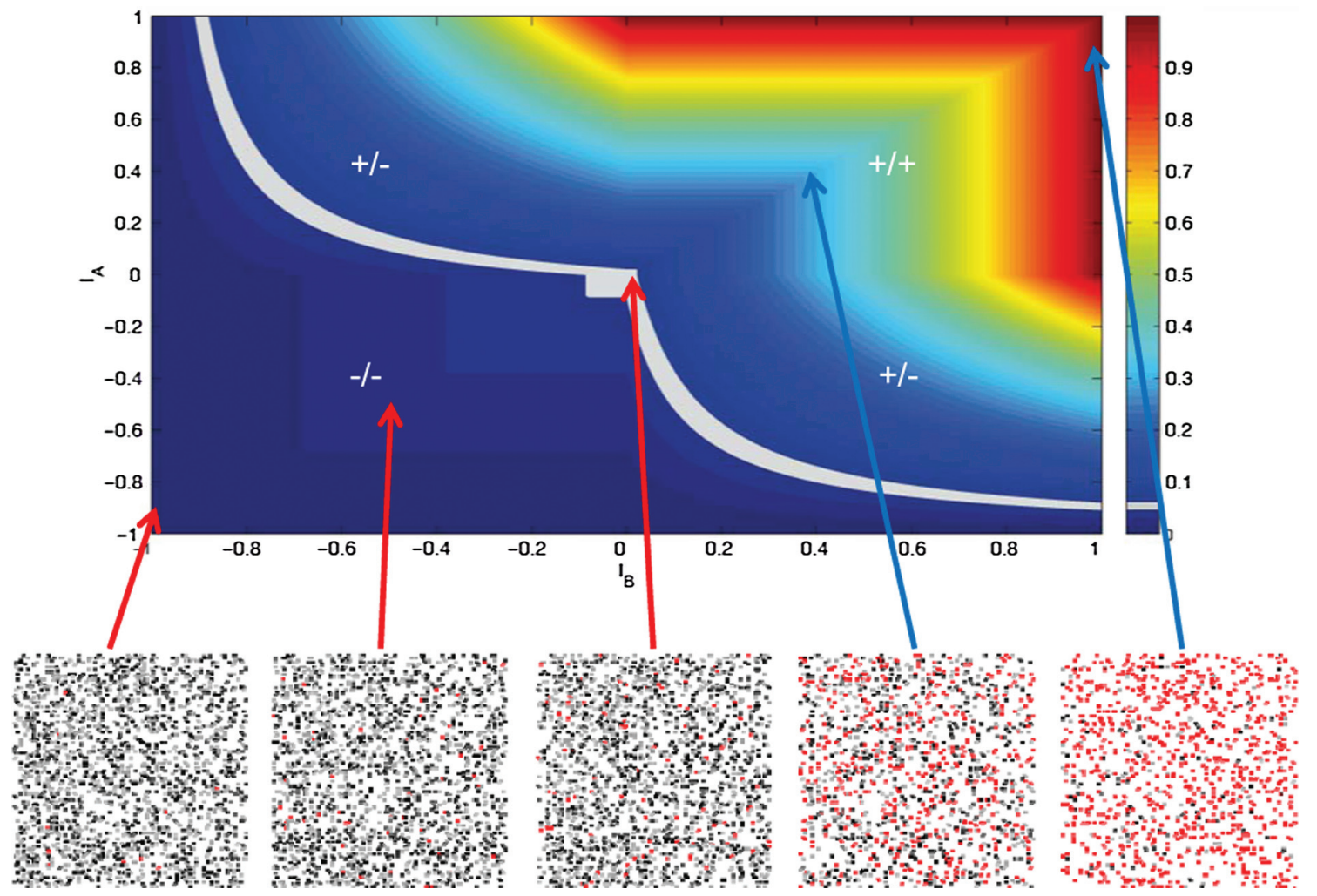

Figure 2. Expected co-occurrence across biotic-interaction space. Colours on the top graph indicate the intensity of the predicted co-occurrence between species A ( $\mathrm{y}$ axis) and B (x axis), where increasing gradients of red indicate increased co-occurrence and increasing gradients of blue indicate decreased co-occurrence. The light gray line indicates the portion of biotic-interaction space where co-occurrence between two species is no different than expected with the null model. The numbers on the $\mathrm{y}$ and $\mathrm{x}$ axes represent interactions (I) of varying signal $(+,-, 0)$ and strength $(\geq 0 \leq 1)$. The lower scatter diagrams provide examples of simulated distributions of species $\mathrm{A}$ (black) and B (gray), with their respective co-occurrence (red), for interactions of varying sign and strength. Both species have prevalence $\rho=0.3$.

branches of science (Levin 1992). Our point-process models offer a novel and general framework for studying the signature of any type of biotic interactions across scales. The results illustrate how relatively simple mathematical models can make testable predictions about species cooccurrence across spatial scales, thus enhancing understanding of community patterns in ecology. Specifically, our findings shed light onto the long-standing controversy of whether the geographical signature of biotic interactions is maintained across spatial scales (Wiens 1989, Schneider 2001). It is typically assumed that the geographical signature of biotic interactions is scale dependent, with climate structuring the broad outlines of species ranges and biotic interactions affecting patterns of local abundances (Whittaker et al. 2001, Pearson and Dawson 2003). Competition is often given as an example of the localized effects of biotic interactions (Connor and Bowers 1987, Whittaker et al. 2001, Pearson and Dawson 2003). Our extensive model simulations support the view that the spatial signature of negative interactions is sensitive to scale, i.e. exclusion by competitors at local scales of resolution tends not manifest at coarser scales. In contrast, we also demonstrate that interactions involving positive dependencies between species, such as mutualism $(+/+)$ and commensalism $(+/ 0)$, are more likely to be manifested across scales of resolution. Consumer-resource interactions, such as predation, herbivory, parasitism, or disease $(+/-)$ can also generate scale-independent patterns of coexistence providing that the dependency of the consumer on the resource is higher than the repulsion of the resource on the consumer; probably a common feature of consumerresource interactions.

Previous studies have suggested that consumer-resource interactions could modify the regional composition of species pools (Ricklefs 1987) and control for species range limits (Hochberg and Ives 1999) and diversity (Jabot and Bascompte 2012). Recent findings also highlighted the disproportionate effects of consumers in shaping local responses of resources to climate change (Post 2012). Our results generalize and extend these inferences. Specifically, we identify circumstances in which biotic interactions are likely to generate scale-invariant patterns of co-occurrence among species. Based on these results we propose a new scaling law: the degree to which the signatures of biotic interactions on local co-occurrences scale up depends on the net effect of the positive dependencies between species. When the net effect of interactions is positive, the spatial effects of such interactions will tend to scale independence. When the net effect of interactions is negative, the spatial effects of interactions will tend to scale dependence.

Even though our simulations suggest that competitive interactions generate local patterns of co-occurrence that 

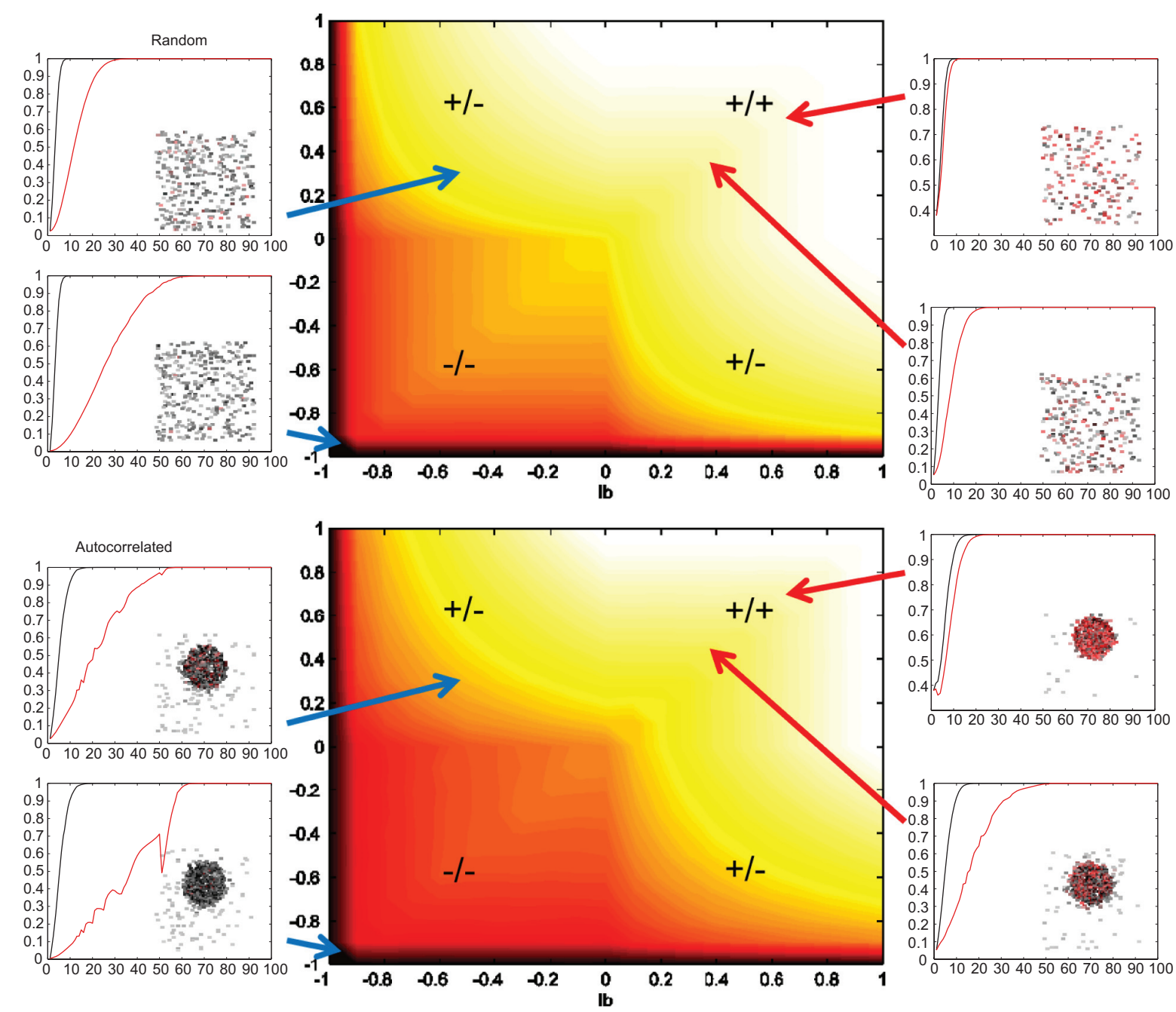

Figure 3. Scale dependence of co-occurrence patterns across biotic interaction space. In the outer scatter plots, red lines indicates 'true' co-occurrence (y axis) between species A and B at increased scales of resolution ( $\mathrm{x}$ axis), while black lines represent estimated co-occurrence after sampling occurrence data at increased scales of resolution. The greater the area between the two curves, the greater the scale dependence in the geographical signatures of biotic interactions. Lattice diagrams are examples of the geographical distributions of species A (black) and B (grey) and their respective overlaps (red) for interactions of varying signal and strength. In the central column of the graphs, increasing gradients of red indicate increased scale dependence (i.e. increased area between red and black lines in outer scatter diagrams), while decreasing gradients of red indicate increased scale independence (i.e. decreased area between red and black lines in outer scatter diagrams): (a) when A and B have prevalence $\rho=0.1$ and both are randomly distributed; (b) when $A$ and B have $\rho=0.1$ and $B$ is geographically structured. Colour scales are log transformed. Estimates of range overlap underlying measurements of scale dependence were obtained with 1000 model runs and values provided are averages across all runs. Summary statistics are provided in Table 1.

do not scale up (for recent empirical evidence of the same pattern see also Segurado et al. 2012), there are circumstances in which the consequences of competition are expected to be manifested at broader scales. Such is the case when competitive exclusion leads to splitting of species ranges at biogeographical extents (Hardin 1960, Horn and MacArthur 1972, Connor and Bowers 1987). To explore this exceptional circumstance we repeated our simulations for the extreme case of repulsion $I_{A}^{-}=1$ and $I_{B}^{-}=1$ (i.e. competition being such that species never co-occur), with highly spatially autocorrelated ranges and subject to varying degrees of range exclusion $\left(0 \leq \mu_{\text {excl }} \leq 1.5\right.$, Supplementary material). With the extremes: 0 representing no enforced range exclusion, potentially leading to checkerboard distributions when ranges are not spatially autocorrelated (the rule used in all previous simulations); and 1.5 representing fully enforced range exclusion leading to range splitting with not edge contact (see supplementary material for more details). We find, as expected, that the greater the degree of exclusion $\left(\mu_{\text {exc }}\right)$ between the ranges of two competing species the greater the degree of scale independence of the resulting geographical patterns (Fig. 4). For example, the area between the curves of the 'sampled' and 'true' co-occurrences when no range exclusion is enforced $\left(\mu_{\text {excl }}=0\right)$ is 82 , while when full range exclusion is enforced $\left(\mu_{\text {excl }}=1.5\right)$ the area between the curves is 77 . These areas between curves are, however, well above mean values across biotic interaction space (Table 1), thus supporting our conclusions regarding strong scale-dependence of the co-occurrence patterns with competition. Whether strong forms of range exclusion have an impact in structuring of regional species pools 
Table 1. Mean and SD (after 1000 repetitions) of scale dependence values across biotic interaction space for mutualism $(+/+)$, competition $(-/-)$, consumer-resource interactions $(+/-)$, commensalism $(+/ 0)$, amensalism $(-/ 0)$. The greater the mean values, the greater the scale dependence of co-occurrence patterns generated by biotic interactions (the larger SD the larger uncertainties). Results are provided for two different prevalence values (10 and 30\%) and for two types of distributions (random and autocorrelated). See Supplementary material Appendix 5, Fig. A1 for a visual representation of these results.

\begin{tabular}{|c|c|c|c|c|}
\hline \multirow{2}{*}{$\begin{array}{l}\text { Prevalence } \\
\text { Distribution }\end{array}$} & \multicolumn{2}{|r|}{$10 \%$} & \multicolumn{2}{|r|}{$30 \%$} \\
\hline & random & autocorrelated & random & autocorrelated \\
\hline \multicolumn{5}{|l|}{$+/+$} \\
\hline Mean & 0.3414 & 1.0758 & 0.1000 & 0.1203 \\
\hline $\mathrm{SD}$ & 0.5188 & 1.3736 & 0.1390 & 0.1627 \\
\hline \multicolumn{5}{|l|}{$-/-$} \\
\hline Mean & 29.5011 & 35.4663 & 21.7188 & 21.9117 \\
\hline SD & 32.9402 & 29.0701 & 36.9765 & 36.9117 \\
\hline \multicolumn{5}{|l|}{$+/-$} \\
\hline Mean & 12.5640 & 15.6700 & 11.0573 & 11.1537 \\
\hline SD & 28.1500 & 26.6214 & 28.9997 & 28.7860 \\
\hline \multicolumn{5}{|l|}{$+/ 0$} \\
\hline Mean & 0.8284 & 2.2766 & 0.2235 & 0.2634 \\
\hline $\mathrm{SD}$ & 0.9412 & 2.2639 & 0.2295 & 0.2646 \\
\hline \multicolumn{5}{|l|}{$-/ 0$} \\
\hline Mean & 19.5134 & 26.1670 & 12.3791 & 12.5908 \\
\hline SD & 26.2082 & 23.5428 & 28.5864 & 28.3371 \\
\hline
\end{tabular}

partly depends on the degree to which they are a common feature at biogeographical scales; this question is beyond the scope of our discussion (but see Connor and Bowers 1987).

Our results have important implications for predictions of the effects of environmental changes on species distributions. For example, microcosms experiments have demonstrated that models of species responses to climate change that ignore competition and parasitoid-host interactions could lead to serious errors (Davis et al. 1998). However, our results suggest that errors arising from discounting the effects of competition would unlikely scale up to biogeographical scales (see also Hodkinson 1999). In contrast, models failing to account for strong positive dependencies between species would likely exclude mechanisms affecting species ranges across a range of scales. Consistent with our prediction, studies have shown that mutualism (Callaway et al. 2002), commensalism (Heikkinen et al. 2007), predation (Wilmers and Getz 2005), herbivory (Post 2012) and parasitism (Araújo and Luoto 2007) could significantly affect species ranges and/or species responses to climate change. If predictions of our models are correct, the bad news is that accurate predictions of climate change effects on species distributions would require the development of more complex models that include biotic interactions (Fordham et al. 2013). The good news is that only a subset of all conceivable biotic interactions would likely matter. Since, most interactions between species are likely to be weak and non-obligate (Bascompte 2007, Araújo et al. 2011), and species with strong positive interactions are a subset of a relatively small number of species with strong interactions, the critical question would then be to identify the species with properties that are capable of affecting distributions and coexistence across scales (see also Gilman et al. 2010). The task of identifying such species is of daunting magnitude, but is less so than documenting and modelling the full web of interactions among species.

\section{Outlook}

We are aware that our models can raise scepticism among empirical and theoretical community ecologists. The standard practice is to predict spatial-population processes from models that explicitly and dynamically account for

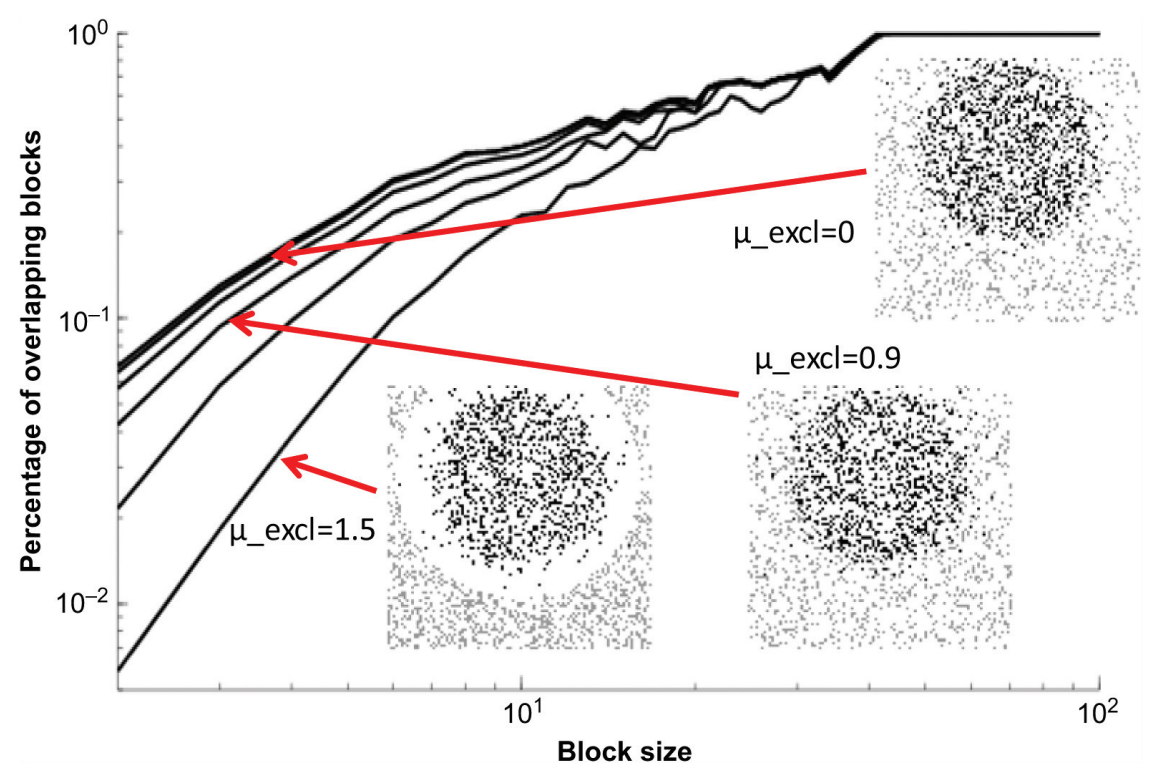

Figure 4. Variation in scale dependence of co-occurrence patterns arising from varying levels of competitive exclusion. With extreme $-I-$ interactions involving $I_{A}^{-}=1$ and $I_{B}^{-}=1$, populations of species A and B never co-occur. So, 'true' co-occurrence is zero (coincident with the $\mathrm{x}$ axis) independently of the size of blocks. By progressively increasing the size of the blocks, sampling leads to classifying species has co-occurring if both species occurred somewhere in the block (black lines). The greater the area between black lines and the horizontal $\mathrm{x}$ axis line the greater the scale dependence of distributional patterns arising from competition. 
consumer-resource interactions. Here, assumptions about these processes are implicit rather than explicit. Instead, we characterize the spatial effects on coexistence of biotic interactions based on the expected attractive and repulsive consequences of these processes. The next step is to test our model predictions through extensive model-model and model-data comparisons. By assuming distributions at steady-state the first comparison that becomes necessary is between expected co-occurrence of species achieved with dynamic Lotka-Volterra models and with static 'pointprocess' models like the ones proposed here. The problem with such comparisons is that consistency with predictions from alternative models lends to conditionally supporting them, but inconsistency leads to inconclusive results as we have no objective way to validate them unless we compare results with data (Oreskes et al. 1994, Araújo and Guisan 2006, Araújo and Peterson 2012). Comparing model results with data is more powerful. However, such tests are difficult to undertake because fully-controlled and fully-replicated experiments at a variety of spatial scales are difficult to undertake and they are extremely costly (Marschall and Roche 1998). Furthermore, our predictions span a full spectrum of biotic interactions rather than focusing on specific types of interaction, thus adding an extra degree of difficulty to experimentation. A possible way forward is to compare predictions from models with microcosm experiments (Livingston et al. 2012). They too have their limitations (Lawton 1998), but a pluralistic approach for testing models is likely the only possible way forward (Kissling et al. 2012).

Acknowledgements - We thank François Guilhaumon, Michael Krabbe Borregaard, and Dominique Gravel for discussion, and Regan Early, Raquel Garcia, and François Guilhaumon for comments on the manuscript. MBA acknowledges the Integrated Program of IC\&DT Call No 1/SAESCTN/ALENT-07-0224FEDER-001755, the Danish NSF, and the Imperial College London's Grand Challenges in Ecosystems and Environments initiative for support of his research. AR is funded through a Portuguese FCT post-doctoral fellowship (SFRH/BPD/ 75133/2010).

The authors contributed equally to this manuscript.

\section{References}

Allen, T. F. H. and Starr, T. B. 1982. Hierarchy: perspectives for ecological complexity. - Univ. of Chicago Press.

Amarasekare, P. 2003. Competitive coexistence in spatially structured environments: a synthesis. - Ecol. Lett. 6: 1109-1122.

Amarasekare, P. et al. 2004. Mechanisms of coexistence in competitive metacommunities. - Am. Nat. 164: 310-326.

Anderson, R. A. et al. 2002. Using niche-based GIS modelling to test geographic predictions of competitive exclusion and competitive release in South American pocket mice. - Oikos 98: 3-16.

Andrewartha, H. G. and Birch, L. C. 1954. The distribution and abundance of animals. - Univ. of Chicago Press.

Araújo, M. B. 2004. Matching species with reserves - uncertainties from using data at different resolutions. - Biol. Conserv. 118: 533-538.
Araújo, M. B. and Guisan, A. 2006. Five (or so) challenges for species distribution modelling. - J. Biogeogr. 33: 1677-1688.

Araújo, M. B. and Luoto, M. 2007. The importance of biotic interactions for modelling species distributions under climate change. - Global Ecol. Biogeogr. 16: 743-753.

Araújo, M. B. and Peterson, A. T. 2012. Uses and misuses of bioclimatic envelope modeling. - Ecology 93: 1527-1539.

Araújo, M. B. et al. 2011. Using species co-occurrence networks to assess the impacts of climate change. - Ecography 34: 897-908.

Araújo, M. B. et al. 2013. Heat freezes niche evolution. - Ecol. Lett. 16: 1206-1219.

Bascompte, J. 2007. Plant-animal mutualistic networks: the arquitecture of biodiversity. - Annu. Rev. Ecol. Evol. Syst. 38: 567-593.

Baselga, A. et al. 2012a. Global patterns in the shape of species geographical ranges reveal range determinants. - J. Biogeogr. 39: 760-771.

Baselga, A. et al. 2012b. Dispersal ability modulates the strength of the latitudinal richness gradient in European beetles. - Global Ecol. Biogeogr. 11: 1106-1113.

Bateman, B. L. et al. 2012. Biotic interactions influence the projected distribution of a specialist mammal under climate change. - Divers. Distrib. 18: 861-872.

Bruno, J. F. et al. 2003. Inclusion of facilitation into ecological theory. - Trends Ecol. Evol. 18: 119-125.

Callaway, R. M. et al. 2002. Positive interactions among alpine plants increase with stress. - Nature 417: 844-848.

Chase, J. M. and Leibold, M. A. 2003. Ecological niches - linking classical and contemporary approaches. - Univ. of Chicago Press.

Cohen, J. E. 1970. A Markov contingency-table model for replicated Lotka-Volterra systems near equilibrium. - Am. Nat. 104: 547-560.

Cohen, J. E. 1971. Estimation and interaction in censored $2 \times 2 \times 2$ contingency table. - Biometrics 27: 379-386.

Colwell, R. K. et al. 2008. Global warming, elevational range shifts, and lowland biotic attrition in the wet tropics. - Science 322: 258-261.

Connell, J. H. 1975. Some mechanisms producing structure in natural communities: a model and evidence from field experiments. - In: Cody, M. L. and Diamond, J. M. (eds), Ecology and evolution of communities. Harvard Univ. Press, pp. 460-490.

Connor, E. F. and Bowers, M. A. 1987. The spatial consequences of interspecific competition. - Ann. Zool. Fenn. 24: 213-226.

Davis, A. J. et al. 1998. Making mistakes when predicting shifts in species range in response to global warming. - Nature 391: 783-786.

Diamond, J. M. 1975. Assembly of species communities. - In: Cody, M. L. and Diamond, J. M. (eds), Ecology and evolution of communities. Harvard Univ. Press, pp. 342-444.

Dieckmann, U. et al. (eds) 2000. The geometry of ecological interactions: simplifying spatial complexity. - Cambridge Univ. Press.

Dobzhansky, T. 1950. Evolution in the tropics. - Am. Sci. 38: 209-221.

Dormann, C. et al. 2007. Methods to account for spatial autocorrelation in the analysis of species distributional data: a review. - Ecography 30: 609-628.

Fordham, D. A. et al. 2013. Adapted conservation measures are required to save the Iberian lynx in a changing climate. - Nat. Clim. Change 3: 899-903.

Gause, G. F. 1934. The struggle for existence. - Willliams and Wilkins.

Gilman, S. E. et al. 2010. A framework for community interactions under climate change. - Trends Ecol. Evol. 25: 325-331. 
Gotelli, N. J. and Graves, G. R. 1996. Null models in ecology. - Smithsonian Inst. Press.

Gotelli, N. J. and McCabe, D. J. 2002. Species co-occurrence: a meta-analysis of J. M. Diamond's assembly rules model. - Ecology 83: 2091-2096.

Gotelli, N. J. et al. 2010. Macroecological signals of species interactions in the Danish avifauna. - Proc. Natl Acad. Sci. USA 107: 5030-5035.

Gravel, D. et al. 2011. Trophic theory of island biogeography. - Ecol. Lett. 14: 1010-1016.

Grinnell, J. 1917. Field tests of theories concerning distributional control. - Am. Nat. 51: 115-128.

Hairston, N. G. et al. 1960. Community structure, population control, and competition. - Am. Nat. 94: 421-425.

Hanski, I. 1998. Metapopulation dynamics. - Nature 396: 41-49.

Hardin, G. 1960. The competitive exclusion principle. - Science 131: 1292-1297.

Heikkinen, R. et al. 2007. Biotic interactions improve prediction of boreal bird distributions at macro-scales. - Global Ecol. Biogeogr. 16: 754-763.

Hickling, R. et al. 2006. The distributions of a wide range of taxonomic groups are expanding polewards. - Global Change Biol. 12: 450-455.

Hochberg, M. E. and Ives, A. R. 1999. Can natural enemies enforce geographical range limits? - Ecography 22: $268-276$

Hodkinson, D. J. 1999. Species response to global environmental change or why ecophysiological models are important: a reply to Davis et al. - J. Anim. Ecol. 68: 1259-1262.

Horn, H. S. and MacArthur, R. H. 1972. Competition among fugitive species in a harlequin environment. - Ecology 53: 749-752.

Horner-Devine, M. C. et al. 2007. A comparison of taxon co-occurrence patterns for macro- and microorganisms. - Ecology 88: 1345-1353.

Humphries, C. J. and Parenti, L. R. 1999. Cladistic biogeography, interpreting patterns of plant and animal distributions. - Oxford Univ. Press.

Hutchinson, G. E. 1957. Concluding remarks. - Cold Spring Harbor Symp. Quant. Biol. 22: 145-159.

Jabot, F. and Bascompte, J. 2012. Bitrophic interactions shape biodiversity in space. - Proc. Natl Acad. Sci. USA 109: 4521-4526.

Kissling, W. D. et al. 2012. Towards novel approaches to modelling biotic interactions in multispecies assemblages at large spatial extents. - J. Biogeogr. 12: 2163-2178.

Kot, M. 2001. Elements of mathematical ecology. - Cambridge Univ. Press.

Law, R. and Dieckmann, U. 2000. A dynamical system for neighborhoods in plant communities. - Ecology 81: 2137-2148.

Lawton, J. H. 1998. Ecological experiments with model systems: the ecotron facility in context. - In: Resetarits Jr, W. J. and Bernanrdo, J. (eds), Experimental ecology: issues and perspectives. Oxford Univ. Press, pp. 170-182.

Legendre, P. 1993. Spatial autocorrelation: trouble or new paradigm? - Ecology 74: 1659-1673.

Leibold, M. A. 1997. Similarity and local co-existence of species in regional biotas. - Evol. Ecol. 12: 95-110.

Lenoir, J. et al. 2008. A significant upward shift in plant species optimum elevation during the 20th century. - Science 320: $1768-1771$

Levin, S. A. 1974. Dispersion and population interactions. - Am. Nat. 108: 207-228.

Levin, S. A. 1992. The problem of pattern and scale in ecology: the Robert H. MacArthur Award lecture. - Ecology 73: 1943-1967.
Livingston, G. et al. 2012. Competition-colonization dynamics in experimental bacterial metacommunities. - Nat. Commun 3: 1234.

Loehle, C. 1998. Height growth rate tradeoffs determine northern and southern range limits for trees. - J. Biogeogr. 25: 735-742.

MacArthur, R. H. 1972. Geographical ecology: patterns in the distribution of species. - Harper and Row.

Madon, B. et al. 2013. Community-level vs species-specific approaches to model selection. - Ecography doi: 10.1111/ j.1600-0587.2013.00127.x

Marschall, E. A. and Roche, B. M. 1998. Using models to enhance the value of information from observations and experiments. - In: Resetarits Jr, W. J. and Bernardo, J. (eds), Experimental ecology: issues and perspectives. Oxford Univ. Press, pp. 281-297.

McGill, B. J. 2010. Matters of scale. - Science 328: 575-576.

McPherson, J. M. et al. 2006. Using coarse-grained occurrence data to predict species distributions at finer spatial resolutions - possibilities and limitations. - Ecol. Model. 192: 499-522.

Meier, E. S. et al. 2010. Biotic and abiotic variables show little redundancy in explaining tree species distributions. - Ecography 33: $1038-1048$.

Nogués-Bravo, D. and Araújo, M. B. 2006. Species richness, area and climate correlates. - Global Ecol. Biogeogr. 15: 452-460.

Oreskes, N. et al. 1994. Verification, validation, and confirmation of numerical models in the earth sciences. - Science 263: 641-646.

Ovaskainen, O. et al. 2010. Modeling species co-occurrence by multivariate logistic regression generates new hypotheses on fungal interactions. - Ecology 91: 2514-2521.

Pearson, R. G. and Dawson, T. E. 2003. Predicting the impacts of climate change on the distribution of species: are bioclimate envelope models useful? - Global Ecol. Biogeogr. 12: 361-371.

Peterson, A. T. et al. 2011. Ecological niches and geographical distributions. - Princeton Univ. Press.

Post, E. 2012. Ecology of climate change. - Princeton Univ. Press.

Pulliam, H. R. 1988. Sources, sinks, and population regulation. - Am. Nat. 132: 652-661.

Rahbek, C. and Graves, G. R. 2001. Multiscale assessment of patterns of avian species richness. - Proc. Natl Acad. Sci. USA 98: 4534-4539.

Ricklefs, R. E. 1987. Community diversity - relative roles of local and regional processes. - Science 235: 167-171.

Ricklefs, R. E. 2010. Host-pathogen coevolution, secondary sympatry and species diversification. - Phil. Trans. R. Soc. B 365: 1139-1147.

Root, T. L. 1988. Environmental factors associated with avian distributional boundaries. - J. Biogeogr. 15: 489-505.

Russell, R. et al. 2006. Scale, environment, and trophic status: the context dependency of community saturation in rocky intertidal communities. - Am. Nat. 167: E158-E170.

Schneider, D. C. 2001. The rise of the concept of scale in ecology. - BioScience 51: 545-553.

Schoener, T. W. 1982. The controversy over interspecific competition: despite spirited criticism, competition continues to occupy a major domain in ecological thought. - Am. Sci. 70: 586-595.

Segurado, P. et al. 2012. Patterns of coexistence of two species of freshwater turtles are affected by spatial scale. - Basic Appl. Ecol. 13: 371-379.

Soberón, J. M. 2010. Niche and area of distribution modeling: a population ecology perspective. - Ecography 33: 159-167.

Svenning, J.-C. and Skov, F. 2004. Limited filling of the potential range in European tree species. - Ecol. Lett. 7: 565-573.

Travis, J. M. J. et al. 2005. The interplay of positive and negative species interactions across an environmental gradient: insights 
from an individual-based simulation model. - Biol. Lett. 1: $5-8$.

Urban, D. L. 2005. Modeling ecological processes across scales. - Ecology 86: 1996-2006.

Veech, J. A. 2006. A probability-based analysis of temporal and spatial co-occurrence in grassland birds. - J. Biogeogr. 33: 2145-2153.

Wallace, A. R. 1878. Tropical nature and other essays. Macmillan.

Walther, G.-R. et al. 2005. An ecological 'footprint' of climate change. - Proc. R. Soc. B 272: 1427-1432.

Supplementary material (Appendix ECOG-00643 at $<$ www.oikosoffice.lu.se/appendix $>$ ). Appendix 1-5.
Whittaker, R. J. et al. 2001. Scale and species richness: towards a general, hierarchical theory of species diversity. - J. Biogeogr. 28: 453-470.

Whittaker, R. J. et al. 2005. Conservation biogeography: assessment and prospect. - Divers. Distrib. 11: 3-23.

Wiens, J. A. 1989. Spatial scaling in ecology. - Funct. Ecol. 3: 385-397.

Wilmers, C. C. and Getz, W. M. 2005. Gray wolves as climate change buffers in Yellowstone. - PLoS Biol. 3: e92.

Woodward, F. I. 1987. Climate and plant distribution. - Cambridge Univ. Press. 